\title{
Acoplamiento de paredes magnéticas en sandwiches de películas delgadas
}

\author{
M.A. RIVERO'1, E. LÓPEZ ${ }^{1}$, C. AROCA ${ }^{2}$, M.C. SÁNCHEZ ${ }^{1}$, P. SÁNCHEZ ${ }^{2}$ \\ ${ }^{1}$ Departamento de Física de Materiales, Facultad de Física (UCM), 28040, Madrid \\ ${ }^{2}$ Departamento de Física, E.T.S.I. Telecomunicación(UPM) 28040, Madrid
}

\begin{abstract}
Se ha estudiado el acoplamiento entre paredes magnéticas en películas tipo sandwich de permalloy/Cu/permalloy, obtenidas por pulverización catódica, de espesores comprendidos entre $2000 \AA / 200 \AA / 2000 \AA$, para evaluar el campo de acoplamiento entre las paredes. Para realizar este estudio se han cortado la muestras de un tamaño lo suficientemente pequeño para conseguir que la energía magnetostática de la muestra sea del mismo orden de magnitud que la energía de la pared magnética. Los resultados obtenidos muestran la posibilidad de conseguir dots magnéticos de tamaños de micrómetros con un comportamiento biestable.
\end{abstract}

Palabras clave: Procesos de imanación, películas delgadas magnéticas.

Domain wall coupling in magnetic thin film sandwiches

Domain wall coupling in thin film sandwiches of permalloy/Cu/permalloy, obtained by sputtering, of thickness from $2000 \AA / 200 \AA / 2000 \AA$ up to $200 \AA / 50 \AA / 200 \AA$ has been studied to determine the coupling field between domain walls. To perform this study samples were cut in a size small enough to obtain a value of magnetostatic energy of the same order than the domain wall energy. Results show the possibility of obtaining magnetic dots with bistable behaviour of microns size.

Keywords: Magnetization processes, magnetic thin films.

\section{INTRODUCCIÓN}

En este trabajo se pretende estudiar el acoplamiento de paredes magnéticas en películas delgadas crecidas por sputtering y formadas por dos capas ferromagnéticas separadas por una no ferromagnética(1). Se ha estudiado la interacción entre las paredes teniendo en cuenta las energías magnetostática, de anisotropía y de canje y suponiendo que la interacción entre las paredes es sólo de origen magnetostático(2). Se han determinado los campos magnéticos de desacoplo para evaluar la energía de interacción entre las paredes. El objetivo de este trabajo es aportar datos que permitan evaluar el campo magnético producido por dos paredes próximas en la capa no magnética comprendida entre ellas con el fin de utilizarlos en microsensores.

\section{PROCEDIMIENTO EXPERIMENTAL}

Para la realización de este trabajo se han obtenido, mediante pulverización catódica en una cámara de tres magnetrones, muestras tipo sandwich formadas por dos capas de permalloy $\left(\mathrm{Ni}_{80} \mathrm{Fe}_{20}\right)$ separadas por una capa de cobre. Los espesores de las muestras obtenidas de permalloy/Cu/permalloy varían entre $2000 \AA / 200 \AA / 2000 \AA$ y $200 \AA / 50 \AA / 200 \AA$

Las muestras se han crecido sobre substratos de corningglass, induciéndoles una anisotropía magnética durante el crecimiento. Para ello se han colocado un par de imanes de ferrita solidarios al portamuestras que crean un campo magnético de $3200 \mathrm{Am}^{-1}$ en el plano de la muestra. Durante el proceso de obtención la temperatura de las muestras se mantienen a una temperatura de $300^{\circ} \mathrm{C}$, al mismo tiempo que giran con respecto al cañón del sputtering para conseguir una mayor homogeneidad del material obtenido.

El espesor de las muestras se controló mediante un resonador de cuarzo en el interior de la cámara de vacío del sistema de pulverización catódica. Posteriormente se comprobaron los espesores midiéndolos con un rugosímetro en un corte realizado por electroerosión de una muestra patrón de cada material.

Una vez obtenidas las muestras se midieron sus ciclos de histéresis por métodos convencionales de inducción a frecuencias bajas y por efecto Kerr magnetoóptico, MOKE, para obtener la energía de anisotropía inducida. En el caso de las películas de menor espesor solo fue posible obtener el ciclo de histerésis por MOKE, debido al poco material que tienen. En la Fig1 se muestran los ciclos de histéresis de un sandwich de espesores $2000 \AA / 200 \AA / 2000 \AA ̊$ en la dirección de la anisotropía inducida y en la dirección perpendicular. A partir del trabajo de imanación en la dirección perpendicular se obtiene el valor de la constante de anisotropía K. Los ciclos de histéresis de las muestras medido por MOKE aparecen invertidos debido al efecto Kerr negativo del níquel. El valor medido de la constante de anisotropía es $\mathrm{K}=180 \mathrm{Jm}^{-3}$.

Posteriormente las muestras se cortaron en forma de cintas perpendiculares a la dirección de la anisotropía inducida, mediante un sistema de electroerosión desarrollado en nuestro laboratorio. El dispositivo de corte consiste en una punta que se desplaza por encima de la superficie de la muestra movida por motores paso a paso controlados por un programa de ordenador. La muestra se mantiene sumergida en un líquido dieléctrico mientras se aplica una diferencia de potencial entre la punta y el material (que debe ser conductor). Los cortes se realizaron de esta manera con objeto de no inducir ninguna anisotropía adicional.

Las muestras así cortadas, cuyas dimensiones son $10 ¥ 0.5 \mathrm{~mm}$, 10x1 mm, 10x1.5 mm, presentan una configuración de dominios con paredes transversales para reducir su energía magnetostática. Utilizando la técnica Bitter y el efecto Kerr se han estudiado la disposición de estas paredes así como su comportamiento bajo la acción de campos magnéticos de distintas configuraciones. 


\section{RESULTADOS Y DISCUSIÓN}

a) Previamente a cada tipo de medida se desimanan las muestras mediante la aplicación de un campo magnético alterno decreciente en sentido longitudinal a la cinta. En todas las muestras desimanadas se observa una disposición de paredes magnéticas en parejas. Las paredes no son rectas sino que se curvan ligeramente tendiendo a acercarse en los bordes como se muestra en la Fig2. Este resultado estaría justificado si en la capa ferromagnética inferior los dominios tuviesen la imanación en sentido opuesto al correspondiente de la capa superior, de modo que disminuya el campo de dispersión producido por los polos magnéticos, reduciendose la energía magnetostática. En las monocapas la diposición de las paredes es equidistante (3).

b) Las muestras desimanadas se colocan dentro de unos carretes Helmholtz situados en el microscopio para observar el comportamiento de las paredes cuando se aplica un campo continuo creciente paralelo a la dirección de la anisotropía. Las paredes no se desplazan hasta que el campo magnético alcanza un cierto valor o campo crítico, $\mathrm{H}_{0}$, como sería esperable. Cuando se alcanza el valor $\mathrm{H}_{0}$, si el campo aplicado tiene sentido contrario al de la imanación en el dominio entre las dos paredes, éstas se mueven en un salto irreversible y desaparecen al chocar entre sí. Por el contrario, si el campo tiene el mismo sentido que la imanación en el dominio entre las paredes, cuando alcanza el valor $\mathrm{H}_{0}$, las paredes se separan en un salto irreversible y después continúan su movimiento de un modo uniforme hasta que se encuentran con una pared de otra pareja, momento en el cual ambas desaparecen (ver Fig3).

Por tanto, el campo crítico $\mathrm{H}_{0}$ está relacionado con el campo de acoplamiento entre las paredes de la capa inferior y la superior. Como se justificó en el apartado anterior, el vector imanación en las capas inferior y superior es de sentido contrario por lo que el movimiento de las paredes superior e inferior tiene sentidos opuestos y hace falta una energía mínima para desenganchar las paredes de las dos capas, que es la energía suministrada por el campo crítico. Una vez realizado el salto irreversible, las paredes estarían desacopladas, de modo que su energía es diferente. En capas de espesores determinados, esta diferencia de energía es suficiente para que el tipo de estructura de pared acoplada y desacoplada sea distinto. Esto se confirma en las paredes de capas de Permalloy $2000 \AA$ en las que su estructura pasa de ser tipo Neel a ser tipo Cross-Tie, una vez que se ha producido el salto (4)(ver Fig 4 a,b,c y Fig 1).

c) Se hace pasar una corriente eléctrica a través de la muestra en forma de cinta para confirmar el punto anterior. El campo magnético creado por la corriente tiene sentido contrario en cada capa de la muestra (Fig 5), por lo que el movimiento de las paredes debe ser en el mismo sentido en ambas capas y, al no desacoplarse las paredes, el campo crítico debería ser nulo. El experimento confirmó que, en este caso, el movimiento de las paredes se inicia para una intensidad de corriente que produce en la superficie de la muestra un campo magnético $\mathrm{H}_{\mathrm{P}}$ mucho menor que el campo critico $\mathrm{H}_{0}$. El campo $\mathrm{H}_{\mathrm{P}}$ que se corresponde con el campo de propagación, es el campo necesario para que las paredes puedan vencer los defectos del material. La diferencia entre ambos campos $\mathrm{H}_{0}$ y $\mathrm{H}_{\mathrm{p}}$ es el campo de acoplo entre las paredes y su medida nos permite obtener el valor de la energía de acoplamiento entre las paredes. Los valores medidos de estos campos son $\mathrm{H}_{0}=250 \mathrm{Am}^{-1} \mathrm{y}$ $\mathrm{H}_{\mathrm{P}}=30 \mathrm{Am}^{-1}$.
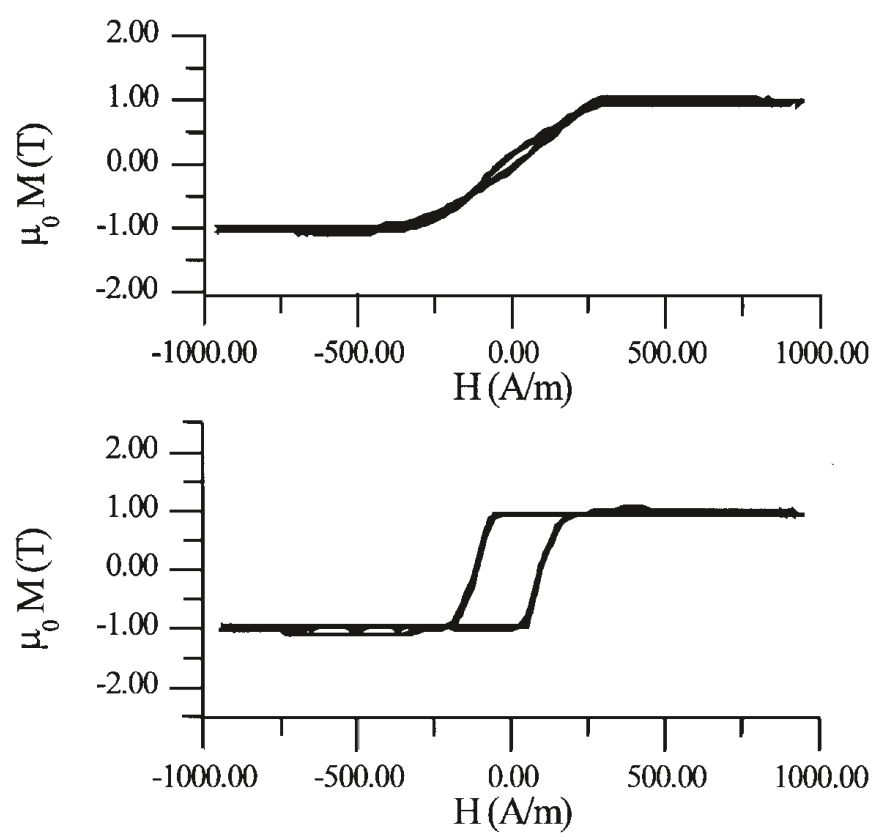

Figura 1.

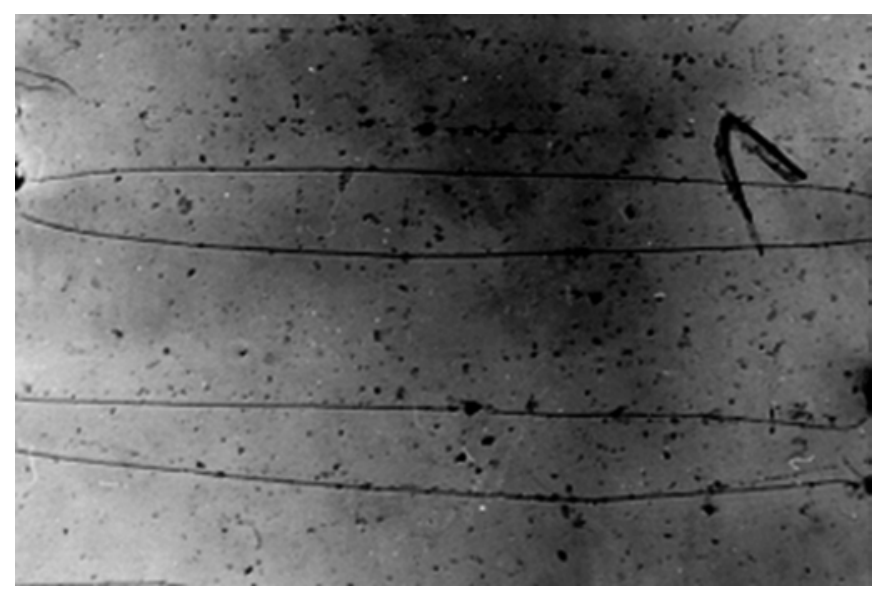

Figura 2. Disposición de las paredes en una muestra desimanada. Se observa la curvatura de las paredes.

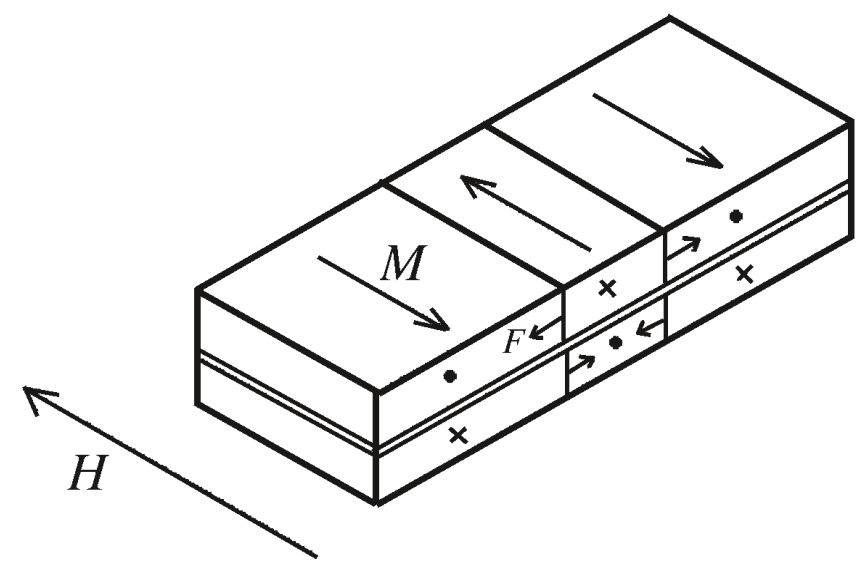

Figura 3. Esquema de a disposición de la imanación y el efecto del campo magnético aplicado. 
a

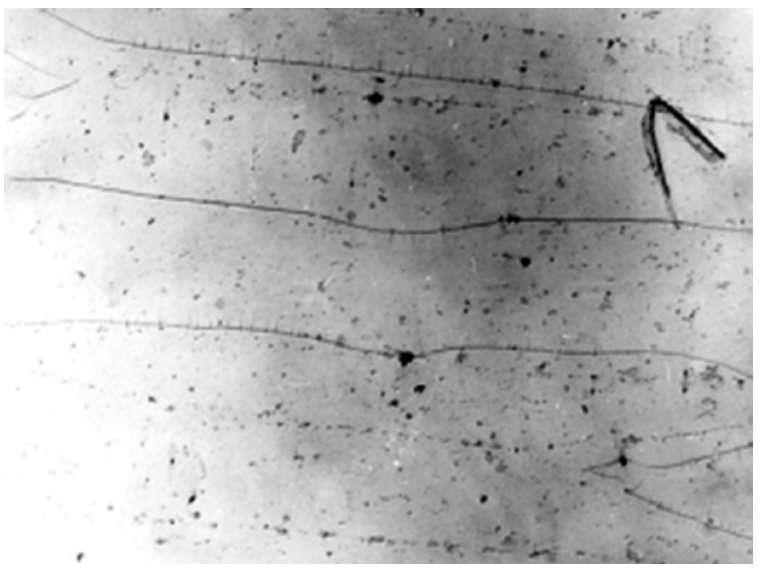

b
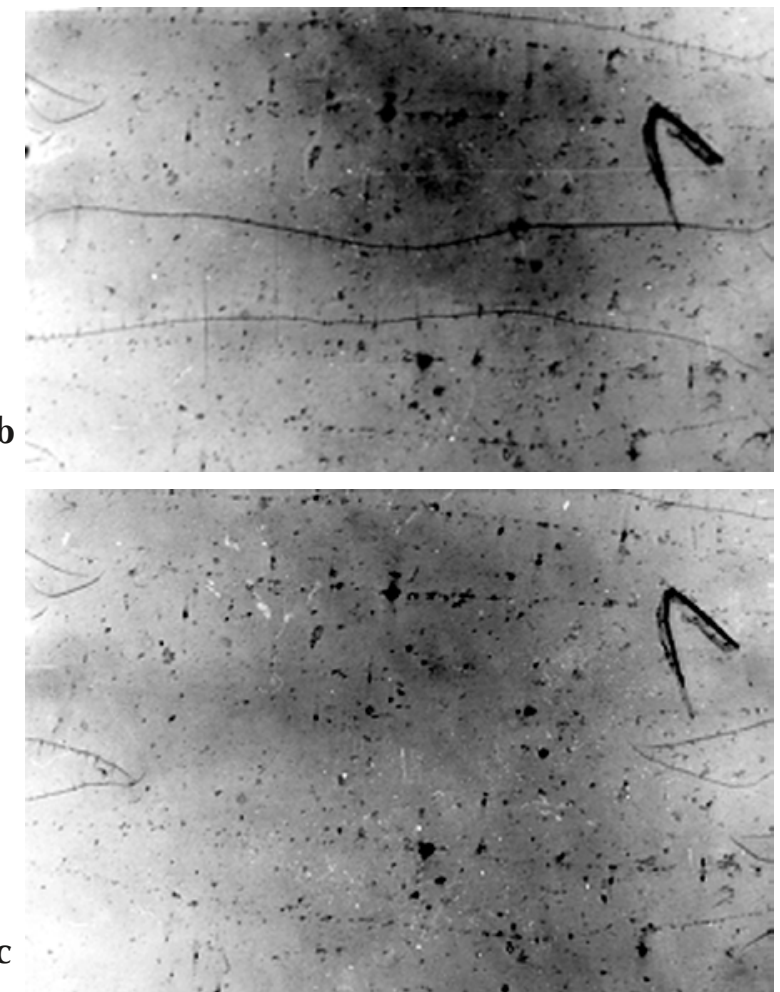

Figura 4. Serie de fotos en las que se observa la situación de las paredes al aplicar un campo magnético creciente. Se observa también el cambio de tipo de pared a comenzar el desplazamiento.

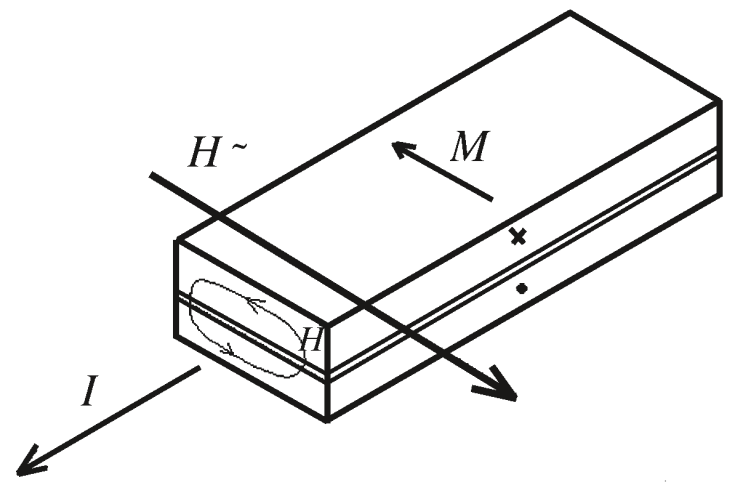

Figura 7. Esquema de la disposición de la imanación al desimanar la muestra al tiempo que se le hace pasar una pequeña corriente.

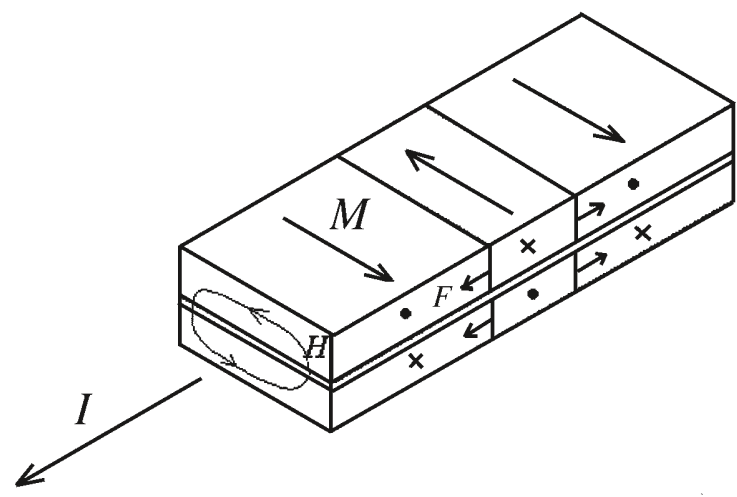

Figura 5. Esquema de la disposición de la imanación y el efecto de una corriente pasando a través de la muestra.

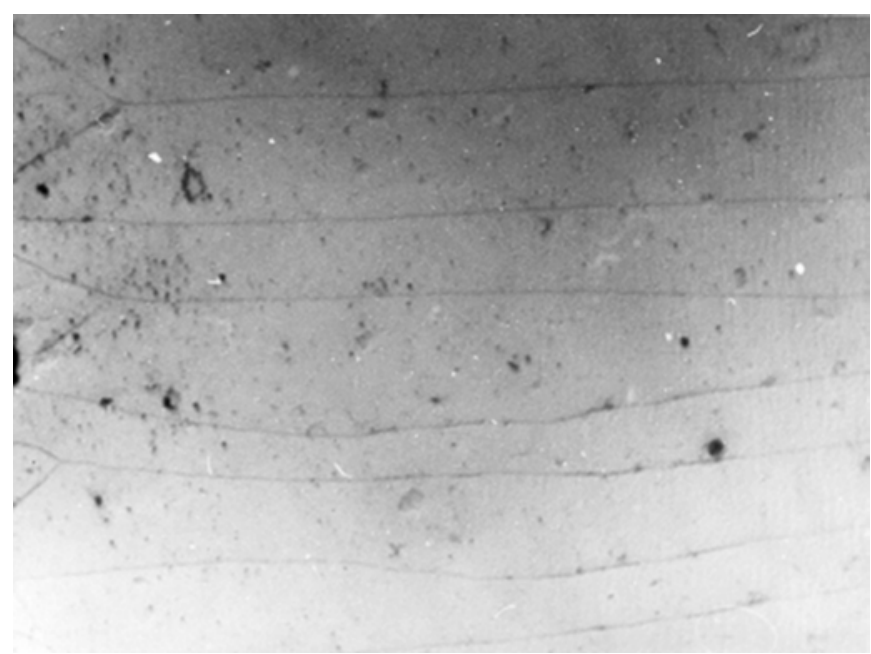

Figura 6. Dominios de cierre en una monocapa de Permalloy de $2000 \AA$.

d) Puesto que la imanación en estas muestras tienen sentido contrario en cada capa, la configuración de la imanación se ve favorecida a cerrarse en el interior de la cinta sin apenas formar polos en la superficie lateral de las muestras. Por otro lado, pese ser la anisotropía baja, no se forman dominios de cierre, como ocurre en las muestras de una sola capa del mismo material y con la misma anisotropía (Fig 6). Además su energía es mucho menor que la suma de las energías de dos monocapas independientes. Todo ello nos sugiere la posibilidad de conseguir monodominios estables en toda una capa.

Esto se comprobó al hacer una desimanación con campo transversal mientras que se hacia pasar una pequeña corriente a través de la muestra lo que favorece la no aparición de paredes (Fig 7). La situación que se observó era la de una cinta sin paredes, por lo que nuestra hipótesis era correcta. En esta situación, si se hace pasar una pequeña corriente en sentido opuesto, aparecía inmediatamente un gran numero de paredes. 

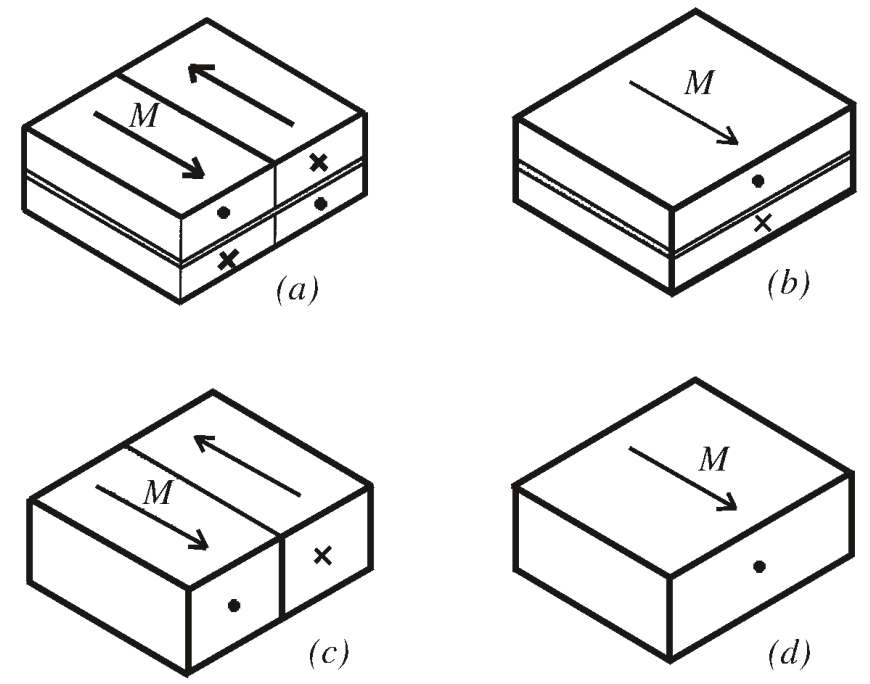

Figura 8. Esquema de las disposiciones de la imanación de los cuadrados para los cuales se realizó la simulación.

\section{CONCLUSIONES}

Este estudio nos ha permitido comprobar la posibilidad de obtener monodominios de tamaño relativamente grande con dos situaciones estables con imanaciones en sentido opuestos nos lleva a pensar en la utilidad de este tipo de configuración para la realización de memorias magnéticas de tipo RAM realizando sistemas en forma de cuadrados en el que se pueda almacenar un bit y que se pueda leer de forma aleatoria.

Se realizo además un estudio mediante simulación magnética en el que se confirmo que la configuración era estable para tamaños de monodominio mucho mayores que en los de materiales formado por una sola capa de material. Se considero un cuadrado de material y se hizo un cálculo de las energías para cada una de las situaciones que se representan en la figura 8. En la figura 9 se representa la energía de cada una de las situaciones con respecto al tamaño del cuadrado. Se observa que el tamaño del monodominio en la bicapa es mucho mayor que en una sola capa(5).

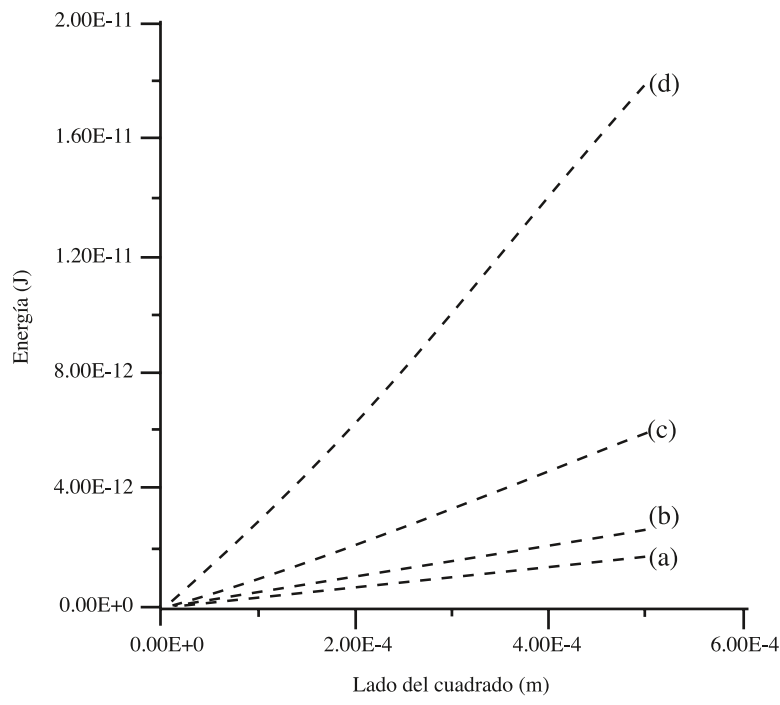

Figura 9. Gráfica en la que se muestra la energía magnetostática en función del tamaño del cuadrado.

\section{AGRADECIMIENTOS}

Este trabajo ha sido financiado por los proyectos MAT971015 y MAT98-0824 de la CICYT.

\section{BIBLIOGRAFÍA}

1. I.B. Puchalska, H.Niedoba, IEEE Trans. Magn. MAG27 3579, (1991).

2. M.Maicas, M.A.Rivero, E. López, C.Aroca, M.C.Sánchez, P. Sánchez, Journal of Magnetism and Magnetic Materials. 289-291 203, (1999) .

3. M.A.Rivero, M.Maicas, E. López, C.Aroca, M.C.Sánchez, P. Sánchez, Journal of Magnetism and Magnetic Materials. 196-197 73, (1999).

4. J. Novak, J. Wenda, L.J. Maksymowicz, J. Magn. Magn. Mat.94 251, (1991).

5. K. Hounadjela,Y. Heny, M. Hehn, L. Piraux $7^{\text {th }}$ E.M.M.A. Conf. Proc. (1998) 116 Figura 1. Ciclos de histéresis por inducción en las direcciones fácil y difícil de una muestra de espesores de capa de $2000 \AA / 200 \AA / 2000 \AA$. 